\title{
Effect of Ground Conductivity on Radiation Pattern of a Dipole Antenna
}

\author{
Md. Shahidul Islam, Md. Shohidul Islam, S. Member, IEEE, Md. Shah Alam
}

\begin{abstract}
This paper aims firstly to develop a theoretical formulation of electromagnetic fields above the earth surface taking into account the finite and infinite conductivity of the ground- which has the advantages of being reasonably fast to compute, secondly to investigate the effect of ground properties on the electric field intensity. For this purpose we have analyzed radiation pattern for finite and infinite ground conductivity, frequencies and different antenna lengths. The monopole is fed to its base. The current distribution along the portion below ground is the image of the current distribution along the original monopole.The image source for calculating electric field, above the infinitely conducting ground surface is just opposite to that of the original source. The image source for calculating electric field above the finitely conducting ground surface is not just opposite to that of the original source. It is affected by the ground properties. For same frequency and identical antenna length but at different ground conductivity, there exists variation in radiation field. It is observed that at small value of ground conductivity the radiation field is small also the direction of radiation field changes. Furthermore it is obtained that rise of frequency increases the radiation field sharply in a particular direction. Finally we can say that at a fixed antenna length, there exists a minimum frequency for measurable radiation field.
\end{abstract}

Index Terms - Antenna, Radiation, Image Current, Dipole Method, Ground Conductivity

\section{INTRODUCTION}

Antennas have become increasingly important to our society. They are everywhere: at our homes, work places, on our cars and aircraft while our ships, satellites and spacecraft bristle with them. Metallic as well as dielectric structures can be designed to launch or radiate waves efficiently into space and to focus or concentrate these waves in a particular direction; these structures are referred to as antennas. They all operate according to the basic principles of electromagnetic. Bandwidth, beam width, polarization are the principal parameters of antenna. An antenna must include impedance matching. When the source matches the load, maximum power is obtained. The antenna must have the ability to match the transmission line.

The antenna must act somewhat as a resonant circuit.The radiation patterns shown so far have been obtained on the assumption that the antenna or antenna array was situated in

Manuscript received on March 12, 2009. This work was supported in part by the Department of Computer Science \& Engineering, RUET and DUET, Rajshahi-6204 and Gazipur-1700, Bangladesh respectively.

Md. Shahidul Islam and Md. Shah Alam are B.Sc Engr. in Electrical and Electronics Engineering, zonal executive of Grameen Phone Limited in Bangladesh.

Md. Shohidul Islam is with the Department of Computer Science \& Engineering in Dhaka University of Engineering \& Technology, Gazipur-1700, Bangladesh. free space far removed from any other conducting bodies or reflecting surfaces.

\section{TERMINOLOGIES}

\section{A. Electric Field}

The area around an electric charge over which the influence of that charge exists is known as electric field.

\section{B. Magnetic Field}

The area around a magnetic pole over which the influence of that pole exists is known as magnetic field.

\section{Electromagnetic Field}

The magnetic field produced by the flow of electricity is known as electromagnetic field.

\section{Radiation Pattern}

A pictorial representation of the directive properties of an antenna's radiation is referred to as its radiation pattern. This pattern is obtained by plotting the magnitude of the far field electric field at a fixed distance from the antenna as a function of $\theta$ and $\phi$. Only the variation of electric field need be plotted as the magnetic field is related to the electric field by $\eta_{0}$ in the far field.

We may determine the form of the waves radiated from an antenna if we know the current distribution over the surface of the antenna. We assume free space and write Maxwell's curl equations for sinusoidal field equation as

$$
\begin{aligned}
& \nabla \times \hat{\mathrm{E}}=-j \omega \mu_{0} \hat{\mathrm{H}} \ldots . . \\
& \nabla \times \hat{\mathrm{H}}=j \omega \varepsilon_{0} \hat{\mathrm{E}}+\hat{J}
\end{aligned}
$$

The term $\hat{J}_{s}$ in equation (2) is a phasor current density in the region, and we will treat it as being the source (know) of the field. We rely on two basic vector identities.

$$
\nabla . \nabla \times \hat{A}=0 \text {. }
$$

Where $\hat{A}$ stands for vector magnetic potential.For any scalar field $\mathrm{V}$, we may write that

$$
\hat{\mathrm{B}}=\nabla \times \hat{\mathrm{A}}
$$

Applying Lorentz condition we have,

$\nabla . \hat{\mathrm{A}}=-j \omega \mu_{0} \varepsilon_{0} \hat{V}$

Using equation (4) magnetic field can be written as

$\hat{\mathrm{H}}=\frac{1}{\mu_{0}} \nabla \times \hat{\mathrm{A}}$ 


\section{MATHEMATICAL FORMULATION}

A. Expression of Electric Field for Infinite Ground conductivity

Electric field is obtained directly from equation (6)

$$
\begin{aligned}
& \hat{\mathrm{E}}=\frac{1}{j \omega \varepsilon_{0}} \nabla \times \hat{\mathrm{H}} \ldots \ldots \ldots \ldots \ldots \ldots . . . \ldots \ldots \\
& \mathrm{E}_{\text {ferfield }}=\frac{j \beta_{0} \hat{I} d l \sin \theta}{4 \pi r} e^{-j \beta_{0} r} a_{\phi}
\end{aligned}
$$

Placing the center of dipole at the origin of the spherical coordinate system as shown in Fig 1.

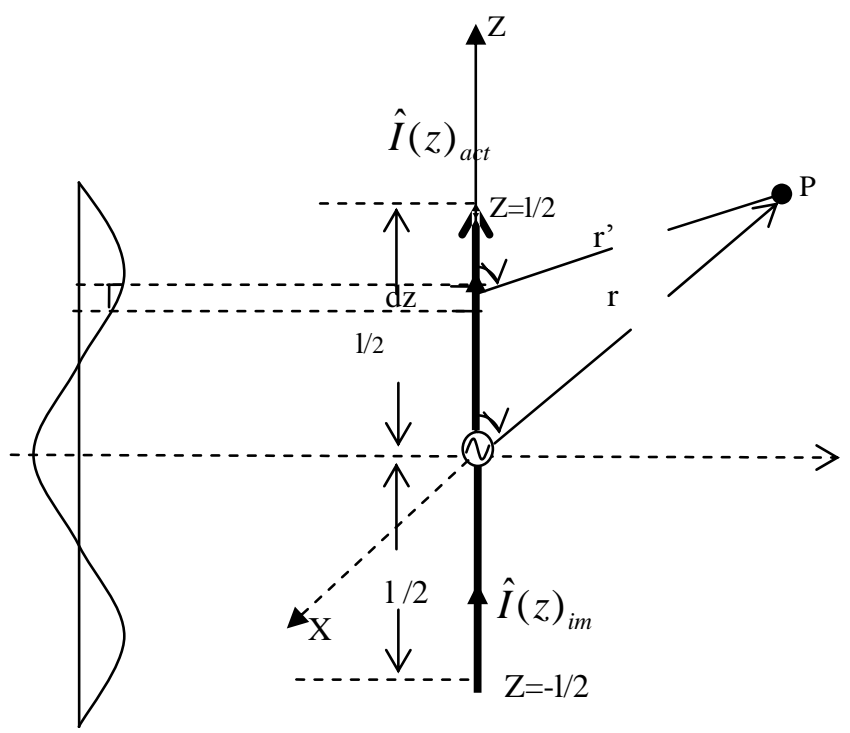

Fig. 1 The long linear dipole antenna

The dipole directed along the $\mathrm{Z}$ axis, we may therefore write an expression for the current distribution along the wire as, considering infinite conductivity, the actual current

$\hat{I}(Z)_{a c t}=\hat{I}_{m} \sin \beta_{0}\left(\frac{l}{2}-Z\right)$.

$0<Z<\frac{l}{2}$

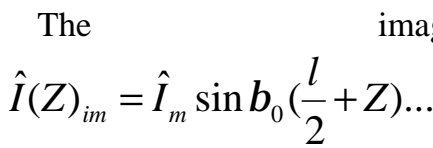
current

$-\frac{l}{2}\langle Z<0$

Consider the infinitesimal segment $\mathrm{dz}$ shown in Fig. 1. If the field point of interest $P$ is located a distance $r$ away from the center of the dipole and angle $\theta$, and a distance $r^{\prime}$ and angle $\theta^{\prime}$ from the current element. The far field electric field in equation (8) due to this element has only a $\theta$ component and a $\frac{1}{r}$ dependence. Thus we have

$$
d E_{\theta}=j \mu_{0} \beta_{0} \frac{\hat{I}_{(z)} \sin \theta^{t}}{4 \pi r^{t}} e^{-j \beta_{0} r^{t}} d z
$$

We are considering only far field, the radiation distance from the center of the dipole at point $\mathrm{p}, r^{\prime}$

we be approximately equal $\left(r \cong r^{\prime}\right)$ and angles $\theta$ and $\theta^{\prime}$ will be approximately equal to $\left(\theta \cong \theta^{\prime}\right)$, as shown in Fig 2.

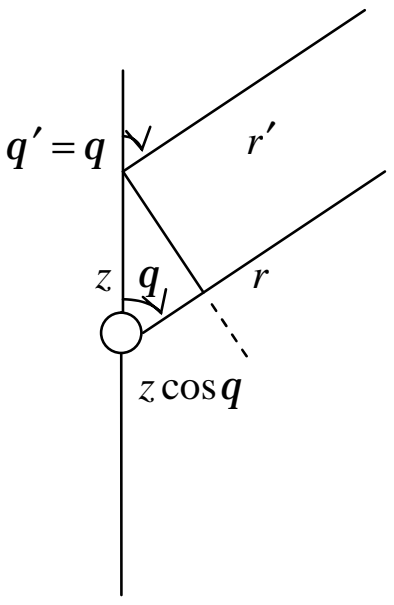

Fig. 2 Far field approximation

We may substitute $r^{\prime}=\mathrm{r}$ into the denominator of equation (11), but we should not substitute this the $e^{-j \beta_{0} r^{\prime}}$ term for the following reason: this term may be written as

$$
e^{-j \beta_{0} r^{\prime}}=\angle-\frac{2 \pi r^{\prime}}{\lambda_{0}}
$$

and its value depends not on the physical distance $r^{\prime}$ but on the electrical distance $\frac{r^{\prime}}{\lambda_{0}}$; Thus, it is not a reasonable approximation to substitute $r^{\prime}$ for $r$ in equation (12).Thus, we assume that the field point is sufficient far, physically, from the antenna.From Fig. 2. We may obtain.

$$
r^{\prime}=r-z \cos \theta
$$

Substituting equation (14) into the phase term in equation (12) and $r$ into the denominator, we obtain

$$
d \hat{E}_{\theta}=j \eta_{0} \beta_{0} \frac{\hat{I}(z) \sin \theta}{4 \pi r} e^{-j \beta_{0}(r-z \cos \theta)} d z
$$

The total electric field is the sum of these contributions- 


$$
\begin{aligned}
E_{\theta}=\int_{z=-\frac{l}{2}}^{z=\frac{l}{2}} j \eta_{0} \beta_{0} \frac{\hat{I}(z) \sin \theta}{4 \pi r} e^{-j \beta_{0} r+j \beta_{0} z \cos \theta} d z & E_{\theta a c t}=\frac{j \eta_{0} I_{m}}{4 \pi r \sin \theta} e^{-j \beta_{0} r} \cdot\left(e^{j \beta_{0} \frac{l}{2} \cos \theta}-\cos \beta_{0} \frac{l}{2}\right. \\
\ldots \ldots \ldots \ldots . . . & \left.-j \cos \theta \cdot \sin \beta_{0} \frac{l}{2}\right) \ldots \ldots \ldots \ldots(22)
\end{aligned}
$$

Integrating equation (15), we obtain

The $\theta$ variation term for actual current of (22) denoted by

$$
\begin{array}{cc}
E_{\theta}=j \frac{\eta_{0} \hat{I}_{m} e^{-j \beta_{0} r}}{4 \pi r} \times 2 . \frac{\cos \left[\beta_{0} \cdot \frac{l}{2} \cdot \cos \theta\right]-\cos \beta_{0} \cdot \frac{l}{2}}{\sin \theta} & F_{\theta a c t}=\frac{1}{\sin \theta}\left(e^{j \beta_{0} \frac{l}{2} \cos \theta}-\cos \beta_{0} \frac{l}{2}\right. \\
& \left.-j \cos \theta \cdot \sin \beta_{0} \frac{l}{2}\right) \ldots \ldots . .(23)
\end{array}
$$

The theta variation term for infinite ground conductivity in equation (16) will be denoted by

$$
F(\theta)=2 \cdot \frac{\cos \left[\beta_{0} \cdot \frac{l}{2} \cdot \cos \theta\right]-\cos \beta_{0} \cdot \frac{l}{2}}{\sin \theta}
$$

Putting, $\beta_{0}=\frac{2 \pi}{\lambda}$

$$
F(\theta)=2 \cdot \frac{\cos \left[\left(\frac{\pi l}{\lambda_{0}}\right) \cos \theta\right]-\cos \left(\frac{\pi l}{\lambda_{0}}\right)}{\sin \theta}
$$

\section{B. Expression of Electrical Field for Actual current}

From the equation (15), we have the far field for actual current $\hat{I}(z) a c t$ is

$$
E_{\theta a c t}=\int_{z=0}^{\frac{l}{2}} \frac{j \eta_{0} \beta_{0} \hat{I}(z)_{a c t} \sin \theta}{4 \pi r} e^{-j \beta_{0}(r-z \cos \theta)} d z
$$

$$
\hat{I}(z)_{a c t}=I_{m} \sin \beta_{0}\left(\frac{l}{2}-z\right)
$$

From equation (19) \& (20) we have,

$$
E_{\theta a c t}=\int_{z=0}^{\frac{l}{2}}\left\{\frac{\dot{j} \eta_{0} \beta_{0} I_{m} \sin \theta}{4 \pi r} e^{-j \beta_{0} r} \cdot e^{j \beta_{0} z \cos \theta} \times\right.
$$

Since,

$$
\hat{I}(z)_{i m}=\left(-\frac{\sigma_{e}}{\varepsilon_{e}}\right) \times\left(\frac{1}{j \omega+\frac{\sigma_{e}}{\varepsilon_{e}}}\right) I_{m} \operatorname{Sin} \beta_{0}\left(\frac{l}{2}+z\right)
$$

$$
\left.\sin \beta_{0}\left(\frac{l}{2}-z\right)\right\} d z
$$

Integrating equation (21), we obtain

Putting $\mathrm{z}=-\mathrm{z}$

From equation (24.1) \& (25.1), we have 


$$
\begin{aligned}
& E(\theta)_{i m g}=\int_{z=0}^{\frac{l}{2}}\left\{\frac{j \eta_{0} \beta_{0} I_{m} \sin \theta}{4 \pi r} e^{-j \beta_{0} r}\left(-\frac{\sigma_{e}}{\varepsilon_{e}}\right)\right. \\
& \left.\times\left(. \frac{1}{j \omega+\frac{\sigma_{e}}{\varepsilon_{e}}}\right) \cdot e^{-j \beta_{0} z \cos \theta} \sin \beta_{0}\left(\frac{l}{2}-z\right)\right\} d z
\end{aligned}
$$

Integrating equation (26) we have,

$$
\begin{aligned}
& E(\theta)_{i m g}=\frac{j \eta_{0} I_{m}}{4 \pi r} e^{-j \beta_{0} r} \cdot\left(-\frac{\sigma_{e}}{\varepsilon_{e}}\right)\left(\frac{1}{j \omega+\frac{\sigma_{e}}{\varepsilon_{e}}}\right) \times \\
& \frac{1}{\sin \theta}\left(e^{-j \beta_{0} \frac{l}{2} \cos \theta}-\cos \beta_{0} \frac{l}{2}+\right.
\end{aligned}
$$$$
\left.j \cos \theta \cdot \sin \beta_{0} \frac{l}{2}\right)
$$

Real part of equation

$\operatorname{Re}\left(F(\theta)_{a c t}\right)=\frac{a}{\operatorname{Sin} \theta}$

The $\theta$ variation term for image current is denoted by

$$
\begin{aligned}
& F(\theta)_{i m g}=\left(-\frac{\sigma_{e}}{\varepsilon_{e}} \cdot \frac{1}{j \omega+\frac{\sigma_{e}}{\varepsilon_{e}}}\right) \cdot \frac{1}{\sin \theta} \times \\
& \left(e^{-j \beta_{0} \frac{l}{2} \cos \theta}-\cos \beta_{0} \frac{l}{2}+j \cos \theta \cdot \sin \beta_{0} \frac{l}{2}\right)
\end{aligned}
$$

$$
\begin{aligned}
& \text { Putting } \quad \beta_{0}=\frac{2 \pi}{\lambda} \\
& F(\theta)_{i m g}=\frac{1}{\sin \theta}\left[K\left\{\cos \left(\frac{\pi l}{\lambda} \cdot \cos \theta\right)-\operatorname{cso}\left(\frac{\pi l}{\lambda}\right)\right\}+\right. \\
& \left.L\left\{\sin \left(\frac{\pi l}{\lambda} \cdot \cos \theta\right)-\cos \theta \cdot \sin \left(\frac{\pi l}{\lambda}\right)\right\}\right]+ \\
& \frac{j}{\sin \theta}\left[K\left\{\cos \theta \cdot \sin \left(\frac{\pi l}{\lambda}\right)-\sin \left(\frac{\pi l}{\lambda} \cdot \cos \theta\right)\right\}+\right. \\
& \left.L\left\{\cos \left(\frac{\pi l}{\lambda} \cdot \cos \theta\right)-\operatorname{cso}\left(\frac{\pi l}{\lambda}\right)\right\}\right] \\
& F(\theta)_{i m g}=\frac{1}{\sin \theta}[(K a+L b)+j(L a-K b)]
\end{aligned}
$$

$$
b=\sin \left(\frac{\pi l}{\lambda} \cdot \cos \theta\right)-\cos \theta \cdot \sin \frac{\pi l}{\lambda}
$$

\section{Expression of Total Electric Field for Finite Ground Conductivity}

The total electric field for finite ground conductivity:

$E(\theta)=E(\theta)_{a c t}-E(\theta)_{i m g}$

$E(\theta)=$

$\frac{j \eta_{0} I_{m}}{4 \pi r \sin \theta} e^{-j \beta_{0} r} \cdot\left(e^{j \beta_{0} \frac{l}{2} \cos \theta}-\right.$

$\left.\cos \beta_{0} \frac{l}{2}-j \cos \theta \cdot \sin \beta_{0} \frac{l}{2}\right) \times$

$\frac{j \eta_{0} I_{m}}{4 \pi r} e^{-j \beta_{0} r} \cdot\left(-\frac{\sigma_{e}}{\varepsilon_{e}}\right)\left(\frac{1}{j \omega+\frac{\sigma_{e}}{\varepsilon_{e}}}\right) \cdot \frac{1}{\sin \theta} \times$

$\left(e^{-j \beta_{0} \frac{l}{2} \cos \theta}-\cos \beta_{0} \frac{l}{2}+j \cos \theta \cdot \sin \beta_{0} \frac{l}{2}\right)$

The $\theta$ variation term for total electric field in (33) denoted by:

$$
F(\theta)=\frac{1}{\sin \theta}[\{a(1-K)-L b\}-j\{b(1+K)-L a\}]
$$

Here,

$a=\cos \left(\frac{\pi l}{\lambda} \cdot \cos \theta\right)-\cos \left(\frac{\pi l}{\lambda}\right) \quad$ and $b=\sin \left(\frac{\pi l}{\lambda} \cdot \cos \theta\right)-\cos \theta \cdot \sin \frac{\pi l}{\lambda}$

\section{ANALYSIS}

In this paper electromagnetic fields have been analyzed yumerically using the theory developed in the previous chapter. The typical model of sinusoidal current is used in analyzing electromagnetic fields. Numerical results using (31) newly developed dipole method are shown for the following parameters such as: conductivity of the ground $=.001 \mathrm{mho} / \mathrm{m}, .01 \mathrm{mho} / \mathrm{m}, 0.1 \mathrm{mho} / \mathrm{m}, 1.0 \mathrm{mho} / \mathrm{m}$, and $10 \mathrm{mho} / \mathrm{m}$; relative permittivity of the ground $\varepsilon_{r}=7$ is used 'when earth is dry'. The effect of ground conductivity on the field pattern of linear center fed monopole antenna 
have been shown in the following figures.Three different antenna length have been considered $\left(l=\frac{\lambda}{2}, \lambda\right.$, $3 \frac{\lambda}{2}$ ). Since the amplitude factor is independent of antenna length, only the relative field patterns as given by the pattern factor have been compared.In figure $3(\mathrm{a})$ to $3(\mathrm{~h})$.

\section{A. Radiation pattern for antenna length $l=\frac{\lambda}{2}$}

Fig. 3 and Fig. 4 are the radiation pattern for actual current and Image current respectively for infinite ground conductivity.

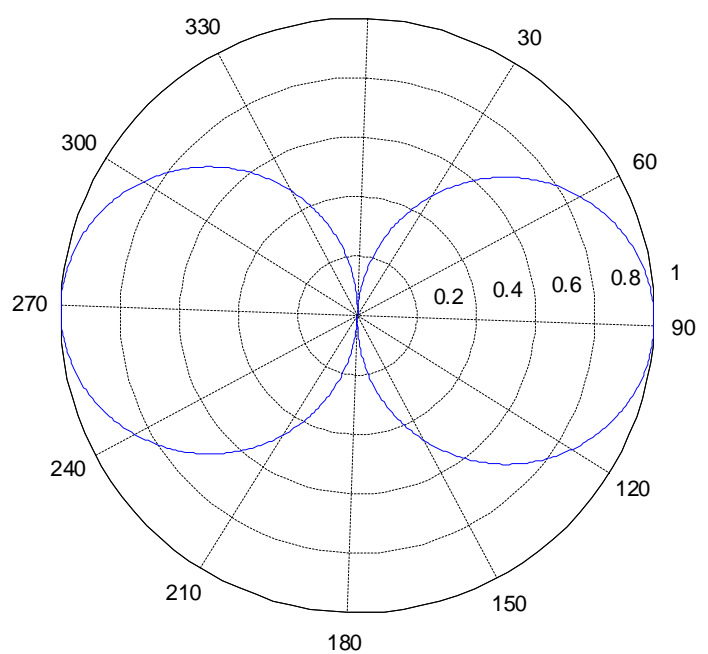

Fig. 3 Radiation pattern due to actual current

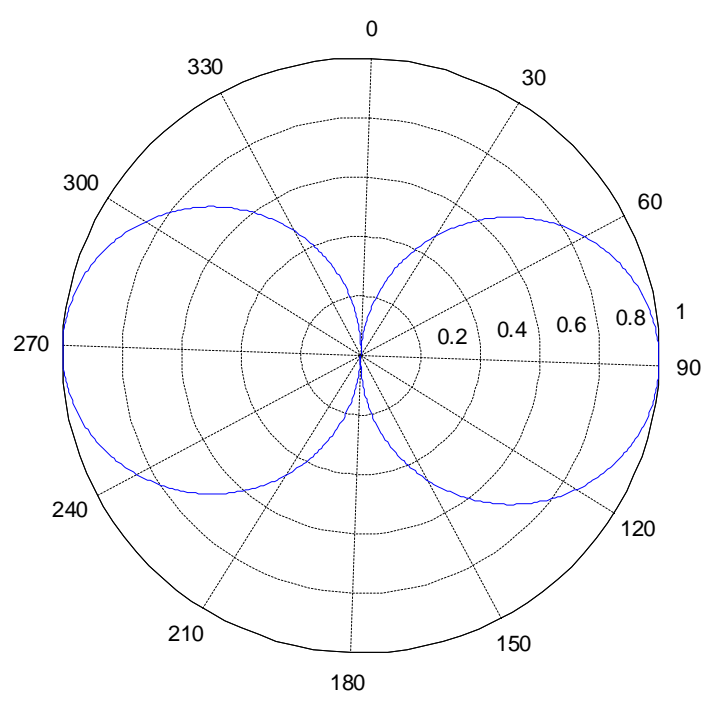

Fig. 4 Radiation pattern due to image current

B. Radiation pattern for antenna length $l=\lambda$

Fig. 5 and Fig. 6 are the radiation pattern for actual current and Image current respectively for infinite ground conductivity.

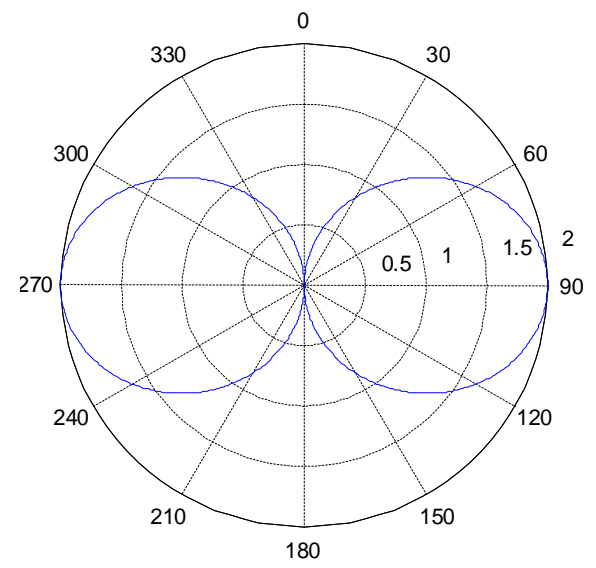

Fig. 5 Radiation pattern due to actual current

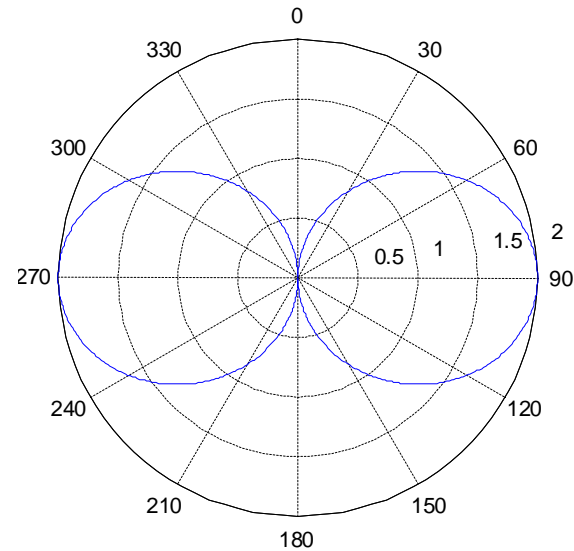

Fig. 6 Radiation pattern due to image current

C. Radiation pattern for antenna length $l=3 \frac{\lambda}{2}$

Fig. 7 and Fig. 8 are the radiation pattern for actual current and Image current respectively for infinite ground conductivity.

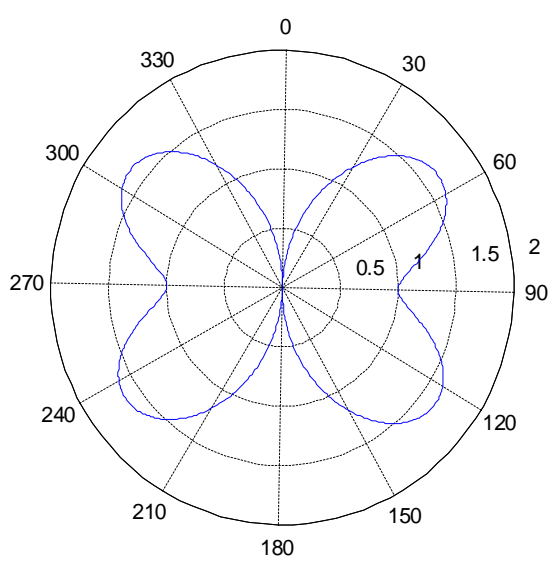

Fig. 7 Radiation pattern due to actual current 


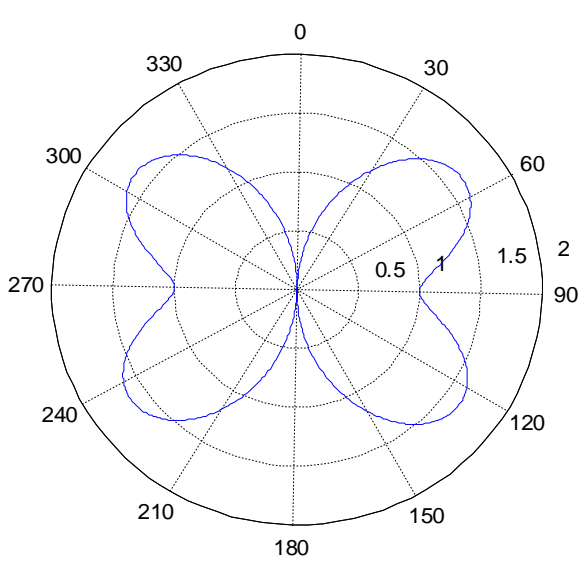

Fig. 8 Radiation pattern due to image current

Fig. 9 and Fig. 10 are radiation pattern for total current and for image infinite current, considering $\sigma=1 \mathrm{mho} / \mathrm{m}$

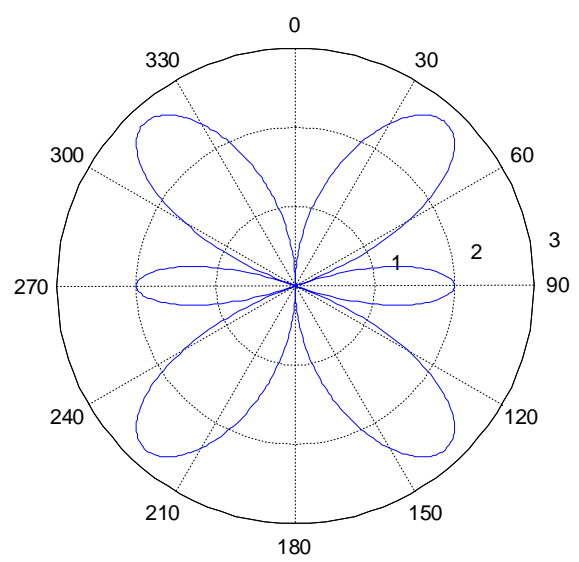

Fig. 9 Radiation pattern due to total current

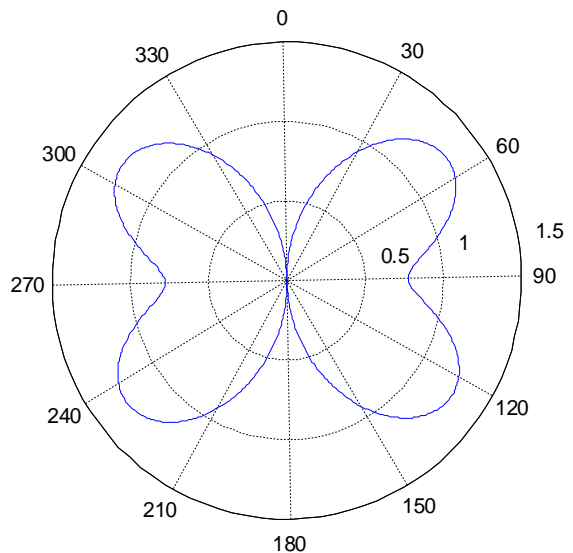

Fig. 10 Radiation pattern due to image infinite current

\section{CONCLUSION}

The effect of ground conductivity on radiation pattern of a dipole antenna has been analyzed. A grounded monopole antenna is excited by sinusoidal source has been considered for this purpose. For the effect of ground conductivity, the radiation field decreases with the decrease of ground conductivity. The image current depends on the ground conductivity and the radiation field is affected by the image current. The image current is also dependent on frequency. For the same wavelength, radiation field increases with the increase of frequency.
From this research we can come to the end that the radiation of antenna can be increased by minimizing the ground effect. Phi $(\phi)$ component of magnetic field intensity can be determined. Using the electric field intensity and magnetic field intensity the average power density vector can be evaluated.

\section{REFERENCES}

[1] Clayton R. Paul, Syed A. Nasar “ Introduction to Magnetic Field” Second Edition.

[2] David K. Cheng "Field and Waves Electromagneties" Second Edition.

[3] Simon Ramo, John R. Whinnery,Theodore Van Duzer "Fields and Waves in Communication Engineering".

[4] Ramakant John D. Kraus, "Antennas". Published by McGRAW-HILL BOOK COMPANY, INC.

[5] Ramakant GEORGE KENNEDY, BERNARD DAVIS, "Electronics Communication System". Published by TATA McGRAW-HILL BOOK COMPANY, LTD.

[6] Ramakant EDWARD C. JORDAN \& KEITH G. BALMAIN "Electromagnetic Waves and Radiating System", Published by Prentice Hall INC.

[7] Magdy F. Iskander, "Electromagnetic Fields and Waves" by Prentice Hall INC

[8] R. Ludwig, A. Konrad, A. D. Joseph, R. H. Katz, "Optimizing the End-to-End Performance of Reliable Flows over Wireless Links",ACM/IEEE MOBICOM'99, Seattle, WA, Aug. 1999.

[9] Li, S. Q. and C. Hwang, "Link Capacity Allocation and Network Control by Filtered Input Rate in High speed Networks", IEEE/ACM Transections on Networking, vol. 3, no. 1, Feb.1995, pp $10-25$. 